\title{
EVALUACIÓN DE GUÍAS DE PRÁCTICA CLÍNICA SOBRE CATARATA
}

\section{ASSESSMENT OF CLINICAL PRACTICE GUIDELINES ABOUT CATARACT MANAGEMENT}

\author{
BLANCO-RIVERA C ${ }^{1}$, GARCÍA-CAEIRO AL ${ }^{2}$, REY-LISTE T ${ }^{2}$
}

\section{RESUMEN}

Propósito: Evaluar la calidad de las guías de práctica clínica (GPC) sobre el manejo de la catarata en adultos.

Material y método: Se realizó una búsqueda bibliográfica seleccionando aquellas GPC basadas en la evidencia o en el consenso de expertos, publicadas a partir del año 2000. Las guías seleccionadas se evaluaron mediante el Instrumento AGREE.

Resultados: Se incluyeron seis guías, tres de ellas resultaron «recomendadas con condiciones o modificaciones», y las otras fueron «no recomendadas». Según los criterios del Instrumento AGREE, ninguna de las guías podría ser recomendada para su uso clínico, si no es sometida previamente a modificaciones.

Palabras clave: Guías de práctica clínica, catarata, AGREE, evidencia clínica, cirugía.

\begin{abstract}
Purpose: To evaluate the quality of clinical practice guidelines (CPG) about the management of cataract in adults.

Material and method: A bibliographic search was carried out selecting the CPG based on the evidence or on the expert consensus, published since the year 2000. The guidelines selected were assessed with the AGREE Instrument.

Results: Six guidelines were assessed. Three were classified as «recommend with provisos or alterations» and the others «would not be recommended». In accordance with the AGREE Instrument criteria, no guidelines can be recommended for their use in the clinical practice if they were not modified previously (Arch Soc Esp Oftalmol 2007; 82: 429-436).
\end{abstract}

Key words: Clinical practice guidelines, cataract, AGREE, clinical evidence, surgery.

\footnotetext{
Recibido: 31/5/06. Aceptado: 12/6/07.

Complejo Hospitalario Universitario de Vigo (CHUVI). Servicio de Oftalmología y Axencia de Avaliación de Tecnoloxías Sanitarias de Galicia (avalia-t) Vigo. Galicia. España.

1 Doctor en Medicina. Complejo Hospitalario Universitario de Vigo (CHUVI).

2 Doctor en Medicina. Axencia de Avaliación de Tecnoloxías Sanitarias de Galicia (avalia-t). Comunicación presentada en el LXXXII Congreso de la S.E.O. (Coruña 2006). 


\section{INTRODUCCIÓN}

Las guías de práctica clínica (GPC) fueron definidas, en el año 1990, por el U. S. Institute of Medici$n e$, como un conjunto de recomendaciones desarrolladas de manera sistemática para ayudar a los clínicos y pacientes en el proceso de la toma de decisiones sobre cuáles son las intervenciones sanitarias más adecuadas para el abordaje de una condición clínica específica. Su utilización ayuda al desarrollo de una práctica clínica correcta y a la obtención de unos mejores resultados en salud. Las GPC ocupan actualmente un papel central en las estrategias de mejora en la toma de decisiones de los sistemas de salud, tanto como herramientas aisladas o incorporadas a programas de gestión clínica de procesos (1).

Los objetivos primordiales de las GPC son, por una parte, mejorar la práctica clínica aumentando, de ese modo, la calidad asistencial y, por otra parte, optimizar también la gestión de los recursos sanitarios (2).

Las características principales que definen una GPC de calidad son, entre otras: estar elaborada por equipos multidisciplinares con alta participación de todos los implicados, estar basada en revisiones sistemáticas de la literatura científica combinadas con el juicio profesional, contener recomendaciones explícitas y clasificadas en función del grado de calidad de la evidencia señalando también aquellas que están basadas en opiniones y, por último, rigor metodológico en su elaboración.

Existen otros instrumentos orientados a ayudar al clínico y que se deben diferenciar de las GPC, son los protocolos y las vías clínicas. Los protocolos son documentos que señalan los pasos a seguir ante un problema asistencial, de forma bastante normativa y sin presentar alternativas. Las vías clínicas se elaboran con el objetivo de ofrecer las distintas directrices que deben seguirse ante determinadas situaciones clínicas, estableciendo en el tiempo las secuencias de cada una de ellas y especificando quién debe realizarlas (3).

Junto con su proliferación hay un déficit en la evaluación de la calidad de las guías y de su idoneidad, existiendo gran cantidad de documentos denominados «guías» que tratando sobre temas similares, presentan inconsistencias y notables discrepancias en sus recomendaciones. La validez y fiabilidad de las guías es especialmente relevante, ya que precisamente las GPC se proponen como un nexo de transferencia rápida entre la investigación y la asistencia, siendo, por tanto, requisito indispensable aparecer como herramientas en las que los clínicos puedan confiar (1). El Instrumento AGREE (Appraisal of Guidelines Research and Evaluation) se desarrolló entre los años 1994 y 1998 en respuesta a múltiples llamamientos realizados a nivel internacional, para establecer una metodología común que promoviera la elaboración rigurosa de GPC y la evaluación de la calidad e impacto en la práctica clínica de las mismas. Se ha demostrado que es un método consistente para evaluar las GPC y su manejo es sencillo (1). Entre sus objetivos se encuentran el desarrollar aproximaciones compatibles para la elaboración de GPC, establecer un modelo para la evaluación y monitorización de las GPC, definir criterios de calidad relevantes para las guías y promover y estimular la difusión de esos criterios mediante intercambios y relaciones de colaboración. El propósito del Instrumento AGREE se podría definir, por tanto, en cuatro puntos: aportar un marco sistemático para la evaluación de la calidad de las GPC y ayudar a quienes elaboran guías a seguir una metodología estructurada y sistemática, a los proveedores de cuidados de salud a evaluar guías antes de adoptar sus recomendaciones en la práctica clínica y, por último, a los encargados de tomar decisiones de diferentes niveles y organismos a recomendar guías para su uso en la práctica (4).

El Instrumento AGREE sirve, tanto para evaluar la calidad de las guías nuevas como la de las ya existentes o sus actualizaciones y consta de 23 ítems distribuidos en 6 áreas, donde cada área intenta abarcar una dimensión diferenciada de la calidad de la guía (Anexo 1). Cada ítem está graduado mediante una escala de 4 puntos: 4 sería «muy de acuerdo», 3 equivale a «de acuerdo», 2 «en desacuerdo» y 1 «muy en desacuerdo». La escala mide la intensidad con que un criterio ha sido cumplido (4).

La necesidad de una GPC se ve justificada cuando se dan determinadas circunstancias en torno a un problema de salud, como una gran variabilidad en su abordaje y que éste represente un gran impacto social y económico. Éstas son circunstancias que se presentan en el manejo de la catarata, que es la principal causa de ceguera en el mundo y que se prevé, su incidencia se duplique en el año 2020 (5). La GPC pretende ayudar al profesional y al paciente a tomar la decisión más adecuada y, al mismo tiempo, a mejorar la gestión de los recursos sanitarios.

El objetivo de este trabajo es evaluar la calidad (mediante la versión española del Instrumento AGREE) (4) de las guías de práctica clínica sobre el 


\section{Anexo 1. Lista de ítems del instrumento AGREE}

A continuación se exponen las áreas que valora el Instrumento AGREE y los criterios que engloba cada una de ellas. Cada criterio equivale a una pregunta que se le hace a la GPC y en base a la respuesta obtenida después de examinar el contenido de la guía, recibirá una puntuación. La puntuación obtenida por cada área (suma de la obtenida por cada criterio) se considera de forma independiente para la valoración global de la guía.

— Área 1 (ítems 1-3): alcance y objetivo de la guía.

Criterio 1: los objetivos generales de la guía están específicamente descritos.

Criterio 2: los aspectos clínicos cubiertos por la guía están específicamente descritos.

Criterio 3: los pacientes a quienes se pretende aplicar la guía están específicamente descritos.

- Área 2 (ítems 4-7): participación de los implicados.

Criterio 4: el grupo que desarrolla la guía incluye individuos de todos los grupos profesionales relevantes.

Criterio 5: se han tenido en cuenta los puntos de vista del paciente y sus preferencias.

Criterio 6: los usuarios de la guía están claramente definidos.

Criterio 7: la guía ha sido probada entre los usuarios diana.

- Área 3 (ítems 8-14): rigor en la elaboración.

Criterio 8: se han utilizado métodos sistemáticos para la búsqueda de la evidencia.

Criterio 9: los criterios para seleccionar la evidencia se describen con claridad.

Criterio 10: los métodos usados para formular las recomendaciones están claramente descritos.

Criterio 11: al formular las recomendaciones han sido considerados los beneficios en salud, los efectos secundarios y los riesgos.

Criterio 12: hay una relación explícita entre cada una de las recomendaciones y las evidencias en las que se basan.

Criterio 13: la guía ha sido revisada por expertos externos antes de su publicación.

Criterio 14: se incluye un procedimiento para actualizar la guía.

- Área 4 (ítems 15-18): claridad y presentación.

Criterio 15: las recomendaciones son específicas y no son ambiguas.

Criterio 16: las distintas opciones para el manejo de la enfermedad o condición se presentan claramente.

Criterio 17: las recomendaciones clave son fácilmente identificables.

Criterio 18: la guía se apoya con herramientas para su aplicación.

- Área 5 (ítems 19-21): aplicabilidad.

Criterio 19: se han discutido las barreras organizativas potenciales a la hora de aplicar las recomendaciones.

Criterio 20: han sido considerados los costes potenciales de la aplicación de las recomendaciones.

Criterio 21: la guía ofrece una relación de criterios clave con el fin de realizar monitorización y/ o auditoría.

- Área 6 (ítems 22-23): independencia editorial.

Criterio 22: la guía es editorialmente independiente de la entidad financiadora.

Criterio 23: se han registrado los conflictos de intereses de los miembros del grupo de desarrollo.

manejo de la catarata en adultos (no se incluyen cataratas congénitas o aquellas secundarias a otras causas).

\section{SUJETOS, MATERIAL Y MÉTODO}

\section{Búsqueda bibliográfica}

Se llevó a cabo una búsqueda bibliográfica en noviembre de 2005 en distintas bases de datos y en las páginas web de los principales organismos internacionales elaboradores/recopiladores de guías de práctica clínica, así como en las páginas gubernamentales y de las sociedades científicas relacionadas con el tema que nos ocupa. En el Anexo 2 se muestran las tablas donde figuran los nombres de las bases de datos y organismos/instituciones en los que se buscó la información, junto con sus direcciones.

\section{Criterios de selección}

De entre los documentos recuperados, se seleccionaron las guías de práctica clínica sobre el manejo de la catarata basadas en la evidencia o en el consenso de expertos, que fueran publicadas a partir del año 2000.

\section{Evaluación de las GPC}

Las guías obtenidas fueron evaluadas con el Instrumento AGREE (versión española) (4), por una especialista en Oftalmología del Complexo Hospitalario Universitario de Vigo (CHUVI) y dos técnicas de la Axencia de Avaliación de Tecnoloxías Sanitarias de Galicia (avalia-t). Previamente las evaluadoras habían participado en talleres sobre el Instrumento AGREE. El proceso se realizó de forma independiente mediante la cumplimentación, por parte de cada evaluadora, de un formulario para 
Anexo 2. Relación de tablas con los nombres de las bases de datos, organismos e instituciones donde se realizó la búsqueda y direcciones electrónicas

\begin{tabular}{ll} 
Guías de Práctica Clínica & Dirección web \\
\hline NGC (National Guidelines Clearinghouse) & http://www.guidelines.gov/ \\
NICE & $\begin{array}{l}\text { http://www.nice.org.uk/ } \\
\text { http://cma.ca/cpgs/ } \\
\text { http://gacguidelines.ca/ }\end{array}$ \\
GNFOBASE & http://www.sign.ac.uk/ \\
SIGN (Scottish Intercollegiate Guidelines Network) & http://www.eguidelines.co.uk/clip \\
CLIP & http://www.nzgg.org.nz/guidelines \\
New Zealand Guidelines Group & http://www.leitlinien.de/english/english/view \\
German Guidelines Clearinghouse & http://www.g-i-n.net/ \\
International Guidelines Network & http://www.chu-rouen.fr/ssf/recomfr.html \\
Répertoire des recommandations de bonne pratique & \\
$\quad \&$ des conférences de consensus francophones & http://www.oqp.med.va.gov/cpg/cpg.htm \\
Department of Veterans Affairs Clinical Practice Guidelines & http://www.iss.gov.co/salud/calidad_guias.htm \\
Instituto de Seguros Sociales de Colombia & http://www.cdf.sld.cu/ \\
Centro para el Desarrollo de la Farmacoepidemiología (Cuba) & http://www.pubgle.com \\
Pubgle & http://www.guiasalud.es \\
guiasalud & http://www.fisterra.es
\end{tabular}

\section{Catálogos de libros}

\section{Dirección web}

Biblioteca Nacional de España

Consejo Superior de Investigaciones Científicas

Locator

British Library

Library of Congress

Organización Mundial de la Salud

CSIC

http://www.bne.es

http://www.csic.es/cbic/cbic.htm

http://locatorplus.gov/

http://www.bl.uk

http://www.loc.gov/catalog/

http://www.who.int/hlt

http://www.cindoc.csic.es

Páginas Gubernamentales

Ministerio de Sanidad y Consumo de España

Health Canada

US National Institute of Health

US Center for Diseases Control

WHO (World Health Organization)

UK Department of Health

\section{Dirección web}

http://www.msc.es

http://www.hc-sc.gc.ca

http://www.nih.gov

http://www.cdc.gov

http://www.who.int

http://www.dh.gov.uk
Sociedades Científicas Temáticas

American Association of Ophthalmology

Royal College of Ophthalmologists

The Royal Australian and New Zealand College

Bases de datos

HTA

MEDLINE ON LINE

EMBASE ON LINE

IBECS (Índice Bibliográfico en Ciencias de la Salud)

Bases de datos ISI

IME (Índice Médico Español)

LILACS ON LINE

DIALNET
Dirección web

http://aao.org

http://www.rcophth.ac.uk

http://www.ranzco.edu
Búsqueda avanzada en motores de búsqueda

GOOGLE

YAHOO

\section{Dirección web}

\section{http://inahta.org}

http://www.ncbi.nlm.nih.gov

http://194.224.36.209:8590

http://bvs.isciii.es/e/bases.php

http://access.isiproducts.com/FECYT

http://bddoc.csic.es:8080/IME/BASIS/ime/web/docu/SF

http://www.bireme.br

http://www.dialnet.unirioja.es 
cada guía. La calidad de la guía en cada una de las 6 áreas de las que consta el Instrumento se determinó mediante el porcentaje del máximo de puntuación posible, tal como recomiendan los autores del AGREE (5). Las puntuaciones de las 6 áreas son independientes y no deben ser agregadas en una única puntuación de calidad. No es probable que una guía puntúe alto en todos los criterios y áreas. El número de criterios altamente valorados y el balance entre áreas deben ayudar a determinar la valoración global de las guías. La evaluación global de las guías se estableció por consenso entre las evaluadoras, siguiendo las recomendaciones del Instrumento AGREE (tabla I).

Los resultados de las valoraciones individuales se volcaron en una tabla excel con la finalidad de obtener las puntuaciones finales, conforme a lo establecido en el Instrumento. Los resultados obtenidos en cada área para cada una de las guías se presentan en el apartado de resultados.

\section{RESULTADOS}

De los resultados de la búsqueda bibliográfica, las GPC que cumplieron los criterios establecidos fueron seis. Los resultados de la evaluación de las mismas son los que se exponen a continuación:

La guía del Royal College of Ophthalmologists (6) se fundamenta en una revisión sistemática de la literatura científica, aunque la búsqueda se realizó únicamente en la base de datos Medline. Las áreas de rigor en la elaboración (área 3) y de claridad y presentación (área 4) son las que obtienen una pun- tuación más alta. En ella se establece una relación explícita entre cada una de las recomendaciones y la evidencia en la que se sustentan, sin embargo, en el grupo de elaboración de la guía no están representadas todas las disciplinas que deberían estar implicadas en el manejo de esta patología. Por otro lado, su puntuación es nula en aplicabilidad y en independencia editorial. Su valoración global sería «recomendada con modificaciones».

La guía de la American Academy of Ophthalmology (7), se basa en una revisión sistemática de la literatura científica, en la que se revisan diferentes bases de datos. Puntúa alto en el área 1 (referida al establecimiento del alcance y objetivos) y en la de rigor en la elaboración (área 3). No alcanza la puntuación del $60 \%$ en el apartado de claridad y presentación, debido fundamentalmente a la dificultad para identificar las recomendaciones clave. Su valoración global sería «recomendada con modificaciones».

En la guía del Ministerio de Salud de Gobierno de Chile (8), la descripción de la metodología de la revisión sistemática no es lo suficientemente explícita para ser reproducible, sí queda claro que se buscó información en diferentes bases de datos de medicina basada en la evidencia. Las recomendaciones establecidas no se asocian a la evidencia en la que se sustentan. El grupo de elaboración es multidisciplinar. En ninguna de las áreas alcanza una puntuación del $60 \%$ y en cuatro de ellas está por debajo del $30 \%$. La valoración global fue «no recomendada».

La guía de la Philippine Academy of Ophthalmo$\operatorname{logy}(9)$, está basada en una revisión sistemática de la literatura científica. Presenta la metodología de elaboración de forma bastante clara pero no especi-

Tabla I. Parámetros orientativos para la valoración global de las guías

\begin{tabular}{|c|c|c|}
\hline Muy recomendada & $\begin{array}{l}\text { La guía puntúa alto ( } 3 \text { ó } 4 \text { ) en la mayoría de los ítems. } \\
\text { La mayor parte de las puntuaciones de las áreas } \\
\text { son superiores al } 60 \% \text {. }\end{array}$ & $\begin{array}{l}\text { Esto indica que la guía tiene una alta calidad } \\
\text { general y que podría ser considerada para su } \\
\text { uso en la práctica sin modificaciones. }\end{array}$ \\
\hline $\begin{array}{l}\text { Recomendada } \\
\quad \text { (con condiciones } \\
\text { o modificaciones) }\end{array}$ & $\begin{array}{l}\text { La guía puntúa alto (3 ó 4) o bajo (1 ó 2) en un } \\
\text { número similar de ítems y la mayoría de las } \\
\text { puntuaciones de las áreas se encuentran entre } \\
\text { el } 30 \text { y el } 60 \% \text {. }\end{array}$ & $\begin{array}{l}\text { Esto indica que la guía tiene una calidad } \\
\text { general moderada. Se considera que } \\
\text { introduciendo algunas modificaciones } \\
\text { (siempre que la información sobre el método } \\
\text { de elaboración esté bien descrito) la guía } \\
\text { puede ser considerada para su uso, especial- } \\
\text { mente cuando no hay otras disponibles sobre } \\
\text { el mismo contenido clínico. }\end{array}$ \\
\hline No recomendada & $\begin{array}{l}\text { La guía puntúa bajo (1 ó } 2 \text { ) en la mayor parte de los } \\
\text { ítems y la mayoría de las puntuaciones de las áreas } \\
\text { son inferiores al } 30 \% \text {. }\end{array}$ & $\begin{array}{l}\text { Esto indica que la guía tiene una baja calidad } \\
\text { general y serias deficiencias. Por tanto, no } \\
\text { debería ser recomendada para su uso en la } \\
\text { práctica. }\end{array}$ \\
\hline
\end{tabular}


fica la fecha de actualización, lo que disminuye su puntuación en el apartado de rigor en la elaboración. Es la única guía que aborda aspectos de monitorización y auditoría y la que obtiene una puntuación más alta en el apartado de claridad y presentación y en independencia editorial. Su valoración global sería «recomendada con modificaciones».

La guía del Alberta Clinical Practice Guidelines Program (10) y de la British Columbia Medical Association (11), están basadas en guías elaboradas por la American Academy of Ophthalmology. Ambas guías alcanzan las puntuaciones más altas en las áreas 1 (alcance y objetivo) y 4 (claridad y presentación). La valoración global de ambas, es, a pesar de todo, de «no recomendadas».

En la tabla II se presentan los resultados obtenidos en cada una de las guías, para cada área, tras su evaluación con el Instrumento AGREE. Las guías que obtienen una mayor puntuación en el área 3 (rigor en la elaboración), fueron la guía del Royal College of Ophthalmologists y la guía de la American Academy of Ophthalmology, siendo dicha puntuación del $63 \%$ en ambos casos.

\section{DISCUSIÓN}

La valoración global de tres de las guías sería, basándonos en las sugerencias expuestas en la tabla
I, «recomendada con modificaciones» ya que la puntuación de la mayoría de las áreas está entre el 30 y 60\%. Según el Instrumento AGREE estas guías, tras las modificaciones pertinentes, podrían ser consideradas para su utilización en la práctica clínica, siempre y cuando no haya otras disponibles de mejor calidad sobre la misma condición (tabla I). Ninguna de las guías evaluadas presenta una relación de preguntas clínicas estructuradas que sirvan como punto de partida de la revisión sistemática y tampoco han sido probadas entre los usuarios diana antes de su implementación. Es importante señalar que todas las guías puntúan bajo en aplicabilidad, debido fundamentalmente, a que en ninguna de ellas existe una relación explícita de las barreras organizativas potenciales a la hora de aplicar las recomendaciones ni se consideran los costes que se podrían derivar de su aplicación en la práctica clínica. Tampoco se ofrece en ningún caso de forma clara una relación de criterios clave para realizar monitorización y/ o auditoría. Se puede concluir, por tanto, que ninguna de las guías evaluadas tiene una alta calidad, según los criterios del AGREE, por lo que en ningún caso se recomendaría su uso en la práctica clínica sin tener que modificarlas.

Las guías con mayor rigor metodológico en su elaboración, y que por tanto podrían ser recomendadas para su utilización (con modificaciones), son las elaboradas por el Royal College of Ophthalmo-

Tabla II. Resultados de la valoración de las guías por áreas

$\begin{array}{cc}\begin{array}{c}\text { Guía del Royal } \\ \text { College } \\ \text { of }\end{array} & \begin{array}{c}\text { Guía de la } \\ \text { American } \\ \text { of Academy }\end{array} \\ \text { Ophthalmologists } & \text { Ophthalmology }\end{array}$

Guía del
Ministerio de
Salud del
Gobierno
de Chile

de Chile

Guía de la
Philippine
Academy
of
Ophthalmology

Guía del
Alberta Clinical
Practice
Guidelines
Program

Guía de la

British

Columbia

Medical

Association

\begin{tabular}{|c|c|c|c|c|c|c|}
\hline $\begin{array}{l}\text { Área } 1 \\
\quad \text { (alcance } \\
\text { y objetivo) }\end{array}$ & $48 \%$ & $70 \%$ & $52 \%$ & $19 \%$ & $52 \%$ & $48 \%$ \\
\hline $\begin{array}{l}\text { Área } 2 \\
\text { (participación } \\
\text { de los implicados) }\end{array}$ & $22 \%$ & $42 \%$ & $11 \%$ & $11 \%$ & $11 \%$ & $14 \%$ \\
\hline $\begin{array}{l}\text { Área } 3 \\
\quad \text { (rigor en } \\
\text { la elaboración) }\end{array}$ & $63 \%$ & $63 \%$ & $22 \%$ & $54 \%$ & $16 \%$ & $10 \%$ \\
\hline $\begin{array}{l}\text { Área } 4 \\
\quad \text { (claridad } \\
\text { y presentación) }\end{array}$ & $64 \%$ & $56 \%$ & $44 \%$ & $75 \%$ & $44 \%$ & $64 \%$ \\
\hline $\begin{array}{l}\text { Área } 5 \\
\quad \text { (aplicabilidad) } \\
\text { Área } 6\end{array}$ & $0 \%$ & $0 \%$ & $0 \%$ & $19 \%$ & $0 \%$ & $7 \%$ \\
\hline $\begin{array}{l}\text { (independencia } \\
\text { editorial) }\end{array}$ & $0 \%$ & $0 \%$ & $0 \%$ & $44 \%$ & $6 \%$ & $22 \%$ \\
\hline
\end{tabular}


logists, la American Academy of Ophthalmology y la Philippine Academy of Ophthalmology.

El Instrumento AGREE ofrece una valoración de la validez de una guía, es decir, la probabilidad de que la guía logre los resultados esperados. Debido a la subjetividad inherente a la evaluación personal de la guía, se recomienda la realización de un taller práctico sobre la utilización del AGREE antes de evaluar la GPC, por el mismo motivo se aconseja, también, la participación de, al menos, tres evaluadores que luego comentarán sus resultados individuales antes de emitir la puntuación definitiva de la guía. La metodología de nuestro trabajo ha seguido rigurosamente estos criterios. Sin embargo, el AGREE no valora el impacto de una guía sobre los resultados en los pacientes ni el contenido clínico de las recomendaciones aplicadas en un contexto local, aspectos que deberían ser igualmente tomados en consideración para realizar un juicio global sobre la utilidad de la misma. Es por esto, que se le da mayor importancia a la hora de seleccionar la GPC a las que puntúan más en el área 3, que es la que valora el rigor de la búsqueda bibliográfica y la metodología seguida por la guía, así como la calidad de sus recomendaciones y la veracidad de las evidencias científicas en que se basan. En nuestro trabajo se han seleccionado las guías que obtuvieron la nota más alta en esta área.

No deja de ser paradójico, que en una patología tan frecuente en nuestro medio, y de tanta importancia en la gestión sanitaria, como es la cirugía de la catarata, no se haya localizado una GPC de alta calidad.

En la situación actual en la que se mueve la profesión médica, es siempre de agradecer el tener unos parámetros de referencia que no sólo ayuden a mejorar la práctica clínica diaria, sino que también ofrezcan una cierta protección desde el punto de vista jurídico en los actos médicos. Por tanto, sería conveniente la elaboración de una GPC sobre el manejo de la catarata en adultos, elaborada con rigor metodológico, a lo que ayudaría sin duda la utilización del Instrumento AGREE.

\section{AGRADECIMIENTOS}

Nuestro más sincero agradecimiento a Dña. Teresa Mejuto Martí por el trabajo de documenta- ción y a la Dra. Teresa Cerdá Mota y al Dr. Severiano Campos García, por la revisión final del manuscrito.

\section{BIBLIOGRAFÍA}

1. Navarro Puerto MA, Ruiz Romero F, Reyes Domínguez, A, Gutiérrez Ibarlucea I, Hermosilla Gago T, Alonso Ortiz del Río $C$, et al. ¿Las guías que nos guían son fiables? Evaluación de las guías de práctica clínica españolas. Rev Clin Esp 2005; 205: 533-540.

2. Grol R, Dalhuijsen J, Thomas S, Veld C, Ruttenb G, Mokkin H. Attributes of clinical guidelines that influence use of guidelines in general practice: observational study. BMJ 1998; 317: 858-861.

3. Woolf SH, Grol R, Hutchinson A ,Eccles M, Grimshaw J. Clinical guidelines: potential benefits, limitations, and harms of clinical guidelines. BMJ 1999; 318: 527530.

4. The AGREE Collaboration. Appraisal of Guidelines for Research \& Evaluation. Instrumento AGREE. Manual de formación; enero 2003 [citado 5 Diciembre 2005]. Disponible en: http://www.agreecollaboration.org/pdf/agreeinstrumentfinal.pdf.

5. Foster A. Cataract and «Vision 2020- the right to sight» iniciative. Br J Ophthalmol 2001; 85: 635-637.

6. The Royal College of Ophthalmologists. Cataract surgery guidelines [citado 7 Diciembre 2005]. Disponible en: http://www.rcophth.ac.uk./docs/publications/CataractsurgeryguidelinesMarch2005Updated.pdf

7. American Academy of Ophthalmology. Cataract in the adult eye [citado 12 Diciembre 2005]. Disponible en: http://www.aao.org/education/library/ppp/loader.cfm?url $=/$ commonspot/security/getfile.cfm\&PageID $=1247$.

8. Ministerio de Salud. Gobierno de Chile. Guía clínica tratamiento quirúrgico de cataratas congénitas y adquiridas [citado 5 Diciembre 2005]. Disponible en: http://www. minsal.cl/ici/guiasclinicas/cataratas.pdf.

9. Philippine Academy of Ophthalmology. Family Medicine Research Group. Clinical practice guideline for the management of cataract among adults [citado 9 Diciembre 2005]. Disponible en: http://www.pao.org.ph/EBO/PDF/ Cataract_CPG_Updated.pdf.

10. Alberta Clinical Practice Guidelines Program. Guideline for surgical \& non-surgical management of cataract in the otherwise healthy adult eye. [citado 12 Diciembre 2005]. Disponible en: http://www.topalbertadoctors.org/TOP/ GPC/Cataracts/Cataracts.htm.

11. British Columbia Medical Association. Guidelines \& Protocols Advisory Committee. Treatment of cataract in adults [citado 9 Diciembre 2005]. Disponible en: http://www.healthservices.gov.bc.ca/msp/protoguides/gps /cataract.pdf. 